\title{
Characterisation of porin genes from Mycobacterium fortuitum and their impact on growth
}

\author{
Soroush Sharbati1,2, Kira Schramm², Sonja Rempel2 ${ }^{2}$ Hwa Wang2, \\ Ronny Andrich ${ }^{2}$, Verena Tykiel ${ }^{3}$, Ralph Kunisch ${ }^{2}$ and Astrid Lewin*2
}

\author{
Address: ${ }^{1}$ Freie Universität Berlin, Institute of Veterinary Biochemistry, Oertzenweg 19b, 14163 Berlin, Germany, ${ }^{2}$ Robert Koch-Institute, Nordufer \\ 20, 13533 Berlin, Germany and ${ }^{3}$ Deutsches Institut für Bautechnik (DIBt) Section II 4 - Health and Environmental Protection, Kolonnenstr. 30 \\ L, 10829 Berlin, Germany \\ Email: Soroush Sharbati - sharbati@zedat.fu-berlin.de; Kira Schramm - schrammk@rki.de; Sonja Rempel - sonjarempel@gmx.de; \\ Hwa Wang - wanghwa@gmx.de; Ronny Andrich - andrichr@rki.de; Verena Tykiel - vty@DIBT.de; Ralph Kunisch - kunischr@rki.de; \\ Astrid Lewin* - lewina@rki.de \\ * Corresponding author
}

Published: 9 February 2009

BMC Microbiology 2009, 9:31 doi:|0.1|86/|47|-2|80-9-3|
Received: I April 2008

Accepted: 9 February 2009

This article is available from: http://www.biomedcentral.com/l47I-2/80/9/3।

(c) 2009 Sharbati et al; licensee BioMed Central Ltd.

This is an Open Access article distributed under the terms of the Creative Commons Attribution License (http://creativecommons.org/licenses/by/2.0), which permits unrestricted use, distribution, and reproduction in any medium, provided the original work is properly cited.

\begin{abstract}
Background: Highly pathogenic mycobacteria like Mycobacterium tuberculosis are characterised by their slow growth and their ability to reside and multiply in the very hostile phagosomal environment and a correlation between the growth rate of mycobacteria and their pathogenicity has been hypothesised. Here, porin genes from M. fortuitum were cloned and characterised to address their impact on the growth rate of fast-growing and pathogenic mycobacteria.

Results: Two genes encoding porins orthologous to MspA from M. smegmatis, porMI and porM2, were cloned from $M$. fortuitum strains, which were originally isolated from human patients. Both porin genes were at least partially able to complement the mutations of a $M$. smegmatis mutant strain lacking the genes $m s p A$ and $m s p C$ with respect to the growth rate. PorMI and porM2 were present in different strains of $M$. fortuitum including the type strain. Comparative expression analysis of porM genes revealed divergent porin expression among analysed $M$. fortuitum strains. Repression of the expression of porins by antisense technique decreased the growth rates of different $M$. fortuitum. The effects of over-expression of por $\mathrm{M} I$ as well as porM2 varied depending on the strain and the concentration of antibiotic added to the medium and indicated that PorMI and PorM2 enhance the growth of $M$. fortuitum strains, but also the diffusion of the antibiotic kanamycin into the cells.
\end{abstract}

Conclusion: This study demonstrates the important role of porin expression in growth as well as antibiotic susceptibility of the opportunistic bacterium $M$. fortuitum.

\section{Background}

Mycobacterium is considered a diverse genus with highly pathogenic members like $M$. tuberculosis or M. leprae as well as less pathogenic, opportunistic and saprophytic species belonging to the so-called rapidly growing myco- bacteria (RGM). The species of RGM able to cause human disease basically belong to the $M$. fortuitum group, the $M$. chelonae/abscessus group and the M. smegmatis group. Members of these groups are commonly seen in aquatic environments like municipal tap water, and health care- 
associated outbreaks are often associated with contact to tap water or water sources such as ice.

The M. fortuitum group includes three taxa: M. fortuitum, $M$. peregrinum and a third biovariant complex. The M. fortuitum group is involved in $60 \%$ of localised cutaneous infections in immunocompetent persons caused by RGM but is a rare cause of pulmonary disease. Most or all of the cases of community-acquired or health care-associated diseases caused by the $M$. fortuitum group are due to $M$. fortuitum. This species basically causes skin lesions, wound infections, postinjection abscesses, postsurgical wound infections or pulmonary disease in previously healthy hosts [1]. Little is known about the virulence mechanisms and persistence of this human pathogen. However, Cirillo et al. [2] and Da Silva et al. [3] reported that M. fortuitum was capable to replicate in amoebae and murine monocytic cells, respectively.

In a previous study, we showed that the intracellular survival of M. smegmatis depended on the amount of porins in the mycobacterial outer membrane (OM). The mutant strain ML10 of M. smegmatis, which lacks the porins MspA and MspC [4], exhibited significantly enhanced intracellular survival compared to the parental strain SMR5 [5]. MspA belongs to a novel class of mycobacterial OM proteins present in many RGM but apparently absent in slowly growing mycobacteria [6]. The main porin of $M$. smegmatis, MspA, is an extremely stable octameric protein composed of $20 \mathrm{kDa}$ monomers [7] and provides the uptake of hydrophilic nutrients across the extraordinarily restricting mycobacterial OM $[7,8]$. By means of DNA hybridisations using a probe derived from the $m s p A$ sequence, Niederweis and colleagues [6] indicated that the genome of $M$. fortuitum contained orthologous porin genes.

Since the saprophytic bacterium M. smegmatis causes disease only in rare cases [1] and shows a very limited intracellular persistence [5], it is important to investigate the role of porins on virulence in pathogenic members of RGM, which are able to multiply intracellularly. M. fortuitum was suggested to be a suitable model Mycobacterium [9]. Like M. tuberculosis, it resides intracellularly in vacuoles restricting interferon- $\gamma$-induced nitric oxide production and limits the maturation of phagosomes [3]. Therefore, $M$. fortuitum was chosen to detect and characterise porins and to analyse their impact first on extracellular growth and in a later stage on intracellular growth. For this purpose, we used two different M. fortuitum strains (10851/03 and 10860/03) that originally were isolated from human patients. Comparative analysis was performed using also the type strain M. fortuitum DSM 46621 .

\section{Results}

In order to verify the taxonomic classification and to define the phylogenetic relationship between the strains analysed, complete sequences of the 16S rRNA genes were determined using the primers, which were described by Adekambi and Drancourt [10]. The phylogenetic analysis of the 16S rRNA sequences confirmed the taxonomic classification of the M. fortuitum strains employed (data not shown).

Comparison of growth rates of the employed strains was performed in broth by measuring the ATP content of the cultures. Compared to other methods for growth measurements such as OD-measurement, cfu-counting or quantification of DNA, the quantification of the ATP-content has the advantage of not being biased by clumping of cultures or by inability of viable bacteria to grow on agar if plated from a broth culture or by occurrence of dead bacteria. We therefore chose this method for the comparison of the growth rates of the three strains. However, the ATP content of bacteria may vary depending on their physiological state and it therefore has to be considered as a surrogate growth marker. As shown in Figure 1 (also see Additional file 1), strain 10851/03 only grew very poorly, while strains 10860/03 and DSM 46621 multiplied strongly from day ten until day 14 or until day 15 , respectively.

\section{PorM genes of M. fortuitum are orthologs of mspA}

To detect porin genes orthologous to mspA in M. fortuitum, preliminary hybridisation experiments were performed with a probe derived from the main porin gene of $M$.

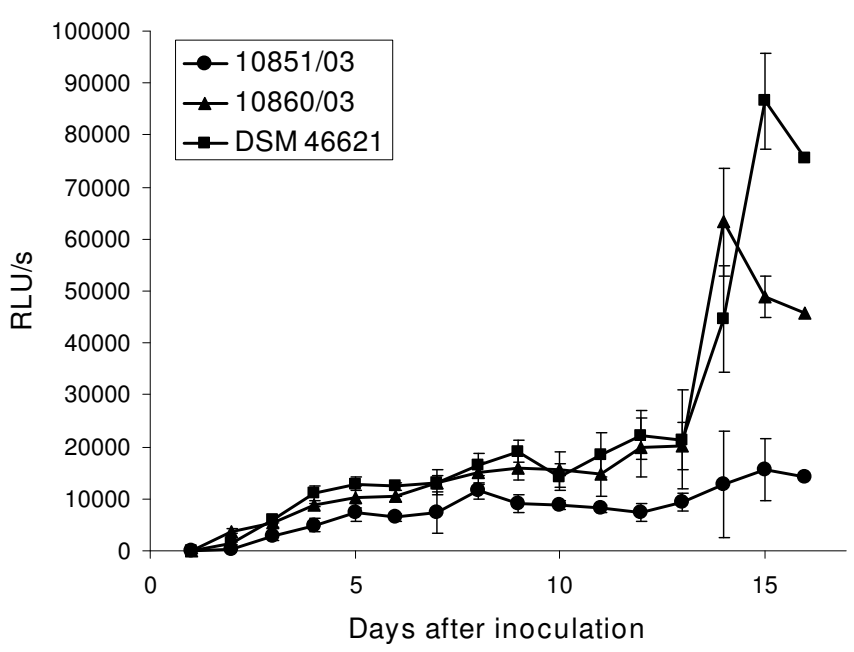

Figure I

Growth rate of the $M$. fortuitum strains $10851 / 03$, $10860 / 03$ and DSM 4662I. The growth rate of the strains was measured by quantification of the ATP-content [displayed as relative light units (RLU)] in broth cultures. 
smegmatis mspA (accession no.: AJ001442) using the primers hpor and npor (Table 1) covering nucleotide 8 to 697. The probe hybridised to the genomic DNA from $M$. fortuitum strains (data not shown). Thus, orthologs seem to exist in all strains analysed.

To clone porin genes from M. fortuitum, genomic DNA of M. fortuitum 10860/03 was digested with SacII, and a 3000 bp fragment, which hybridised to the probe, was cloned in pIV2 and transformed into E. coli. Two clones (pSSp107 and pSSp108) containing porin sequences were isolated. Both plasmids were found to contain the same genomic region of $2895 \mathrm{bp}$, harbouring one porin gene. The inserts were sequenced by primer walking. Both strands of the porin genes and 400 bp of surrounding regions were sequenced at least twice. As shown in Figure
$2 \mathrm{~A}$, the insert of the plasmids contained several open reading frames (ORFs), one of which was an ortholog of $m s p A$. It contained $636 \mathrm{bp}$, encoding a protein of 211 amino acids with an N-terminal signal sequence of 27 amino acids, which was predicted using the SignalP 3.0 Server at http://www.cbs.dtu.dk/services/SignalP/[11]. The in silico analysis of the mature PorM1 (protein without signal peptide) showed a calculated molecular weight of the monomer of $19400 \mathrm{Da}$ and an isoelectric point (pI) of 4.31 .

A hypothetical -10 region of a promoter and a ribosome binding site (RBS) were identified upstream of the coding sequence. Downstream of the ORF a hairpin sequence was detected, which might function as a terminator (Figure 2A). It has to be noted that the sequence similarity between $M$. fortuitum and M. smegmatis was only restricted

Table I: Primers and probes used in this study.

\begin{tabular}{|c|c|c|}
\hline Gene & Primers or probes & Sequence 5'-3' \\
\hline \multirow[t]{2}{*}{$m s p A$} & hpor & 5'-CGG TCT CAG CGA CCG AAC-3' \\
\hline & npor & 5'-CCG GCG ATA CAG TTA GGG A-3' \\
\hline \multirow[t]{2}{*}{ porMI } & $m f-4 I V-f w$ & 5'-TCT CCA GGG GCT GCT TTT G-3' \\
\hline & $m f-4-b w$ & 5'-CGG GAC GCC AAC CAC ATA AC-3' \\
\hline \multirow[t]{2}{*}{ porMI } & komf-3f & 5'-CTG AAG CTT CAC CGA GCT GAG CAT CCT CAC-3' \\
\hline & komf-4b & 5'-GAC ACT AGT CGT TGG CTA CAG AAC AAC ATT CC-3' \\
\hline \multirow[t]{2}{*}{ porM2 } & porM2-5I-fw & 5'-ATG AAG GCA TTC AGT CGG G-3' \\
\hline & porM2-5I-bw & 5'-TGC TCC TCA AAG GAG AAG CG-3' \\
\hline \multirow[t]{2}{*}{ porM2 } & porM2-rev-I & 5'-CGC TTC TCC TTT GAG GAG CA-3' \\
\hline & porM2-rev-2 & 5'-TCC AGA CCC GCA TGA GAT ACG-3 \\
\hline \multirow[t]{2}{*}{ porM2 } & porM2-fw-hind & 5'-ACA AGC TTC AGC AAC GCT GTG AAC GCA-3' \\
\hline & porM2-bw-hpa & 5'-CAG TTA ACA CTA CGG GAC GCT CGT GTC C-3' \\
\hline \multirow[t]{2}{*}{ porM2 } & porM2-rna-fw & 5'-CGC AAG CCT CTT CGT CGG C-3' \\
\hline & porM2-rna-bw & 5'-CCA AGG TGC CCT CGA ACT CAT C-3' \\
\hline \multirow[t]{2}{*}{ porMI } & porMI-as-I & 5'-CGG ATC CTA GGG AGA ACA TGA AGG CAT TCA G-3' \\
\hline & porMI-as-2 & 5'-CGG ATC CTT GTC CAG ACC CGC ATG AGA T-3' \\
\hline \multirow[t]{2}{*}{ porMI } & porMl-fw-hind & 5'-ACA AGC TTG CTC TCA GCC GGT TTT CA-3' \\
\hline & porMI-bw-hpa & 5'-CAG TTA ACG AAC TGG GCG TTC ATG TGC-3' \\
\hline \multirow[t]{2}{*}{ porMI } & porMI-5I-sybr-fw & 5'-GCT GTT TAC GAG CAC GGG C-3' \\
\hline & porMI-5I-sybr-bw & 5'-TTG CGG TCC AGG GGG AAC-3' \\
\hline \multirow[t]{3}{*}{$m s p A$} & mspATaqFW & 5'-CGT GCA GCA GTG GGA CAC CTT-3' \\
\hline & mspATaqBW & 5'-CCA CGA TGT ACT TGG CGC GAC-3' \\
\hline & mspATaqProbe & 5'-FAM-TGG ACC GCA ACC GTC TTA CCC GTG AGT G-TAMRA-3' \\
\hline \multirow[t]{3}{*}{ porM } & mfpqPCRfw & 5'-CGT TCA GCA GTG GGA CAC CTT-3' \\
\hline & mfpqPCRrev & 5'-CCA CGG TGT ACT TGG CCC GGC-3' \\
\hline & mfpqPCRprobe & 5'-FAM-TGG ACC GCA ACC GGC TGA CCC GTG AGT G-TAMRA-3' \\
\hline
\end{tabular}



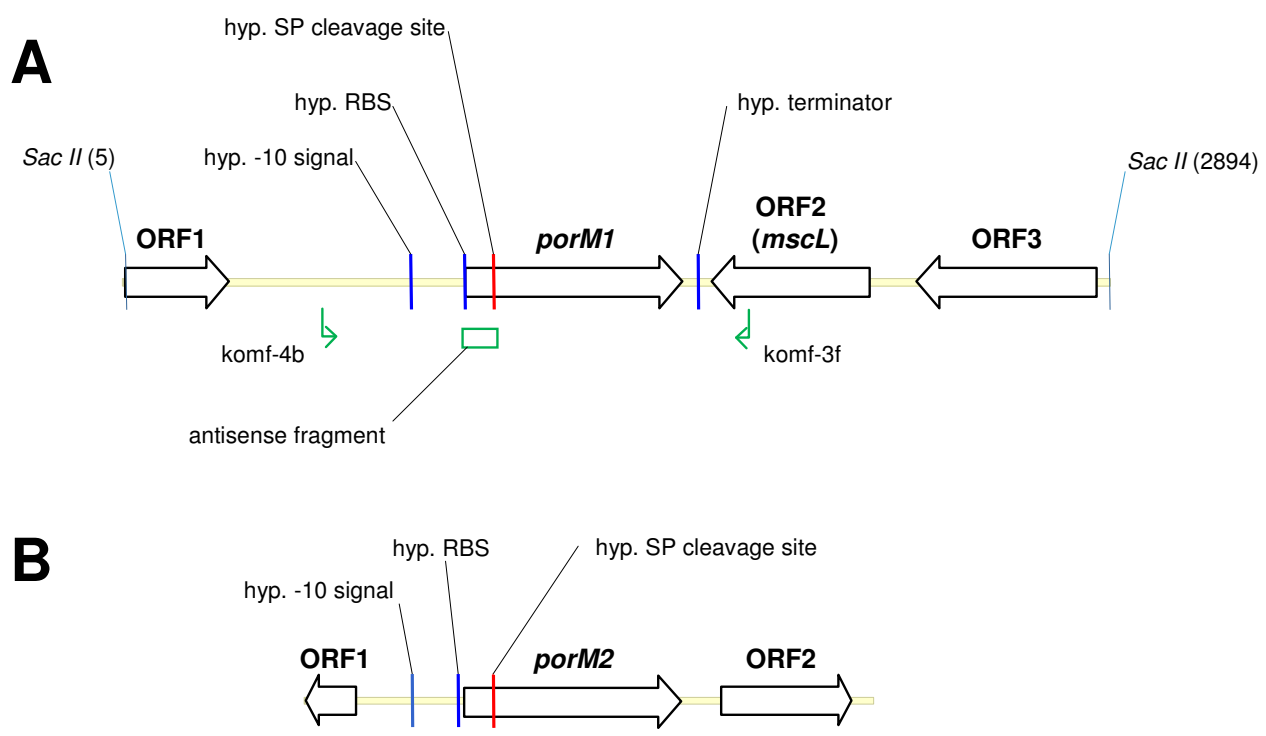

\section{Figure 2}

Map of genomic regions containing porMI from M. fortuitum I 0860/03 and porM2 from M. fortuitum I 085 I/03. Section A shows a 2895 bp region representing the insert of plasmid pSSp 107 . The insert includes the porM I gene and three other ORFs. Up- and downstream to porMI various nucleotide signal sequences were detected: - I0 signal of a promoter (TATGTT), a ribosome binding site (RBS: GGAGA), a signal peptide recognition sequence (SP) of $8 \mathrm{I}$ bp and a hairpin structure, which could represent a terminator. Furthermore, the location of the antisense fragment selected for the generation of plasmid pSRr 106 is indicated. Section B represents a 1697 bp region of M. fortuitum I085I/03 containing porM2 and two other ORFs. Upstream to porM2 a - I0 signal of a promoter (TACGTT), a ribosome binding site (AGGGAGAA) and a signal peptide recognition sequence (SP) of 93 bp were identified. Subsequences were predicted using the software packages MacVector ${ }^{T M}$ 7.2.3 (Accelrys) and Lasergene (DNASTAR).

to the coding sequence. According to the designation of other mycobacterial porin genes and to the instructions of EMBL nucleotide sequence database, the gene was named porM1 (see Table 2 for accession no.).

Besides the porin gene, two other complete ORFs and part of another ORF were detected. ORF1 was interrupted by one of the SacII sites and showed a high similarity to a molybdopterin biosynthesis protein of $M$. tuberculosis CDC 1551 (accession no.: AAK 45260). ORF2 turned out to be a mechanosensitive channel orthologous to the gene mscL from M. avium subsp. paratuberculosis str. 10 (accession no.: NP 959854). ORF3 was similar to the hypothet- ical protein Rv0990c from M. tuberculosis $\mathrm{H} 37 \mathrm{Rv}$ (accession no.: NP 215505). The entire cloned genomic region was blasted against the $M$. tuberculosis genome from the Sanger Institute database http:// www.sanger.ac.uk/cgi-bin/blast/submitblast/

m tuberculosis to examine if the whole region is conserved between $M$. fortuitum and M. tuberculosis. However, only ORF1 and ORF2 possessed nucleotide identities higher than $60 \%$ showing that the region is not conserved among these mycobacteria.

A new probe derived from the porM1 sequence was used to detect porin genes in different $M$. fortuitum strains. The

Table 2: Nucleotide sequence similarity between porMI and porM2 from members of the $M$. fortuitum-group and mspA.

\begin{tabular}{cccc}
\hline Gene & \multicolumn{1}{c}{ Species } & Nucleotide similarity index & Accession-no. to the EMBL nucleotide sequence database \\
\hline \multirow{2}{*}{ porMI } & M. fortuitum DSM 4662I & $88.2 \%$ & $\mathrm{~A} / 880097$ \\
& M. fortuitum 1085I/03 & $88.4 \%$ & $\underline{\mathrm{A} / 880098}$ \\
porM2 & M. fortuitum 10860/03 & $87.4 \%$ & $\underline{\mathrm{A} / 874299}$ \\
& M. fortuitum 1085I/03 & $86.5 \%$ & $\underline{\mathrm{AM} 295792}$ \\
& M. fortuitum 10860/03 & $86.5 \%$ & $\underline{\mathrm{AM} 295793}$ \\
\hline
\end{tabular}


probe hybridised to two fragments of the SacII-digested genomic DNA of different M. fortuitum strains. However, the fragment size differed among different strains (Figure 3 ). Hence, the M. fortuitum genomes contain at least two porin genes.

Next, the presence of porM1 in other M. fortuitum strains was analysed. For this purpose, the porM1-specific primers komf-3f and komf-4b (Figure 2A and Table 1) were chosen to amplify a fragment of approximately $1250 \mathrm{bp}$, comprising the porM1 gene and its flanking regions. PCRs using a polymerase-mix with proofreading activity generated a fragment of the expected size in all strains. Several PCRs were performed and both strands of the different fragments were sequenced. PorM1 was detected in all three $M$. fortuitum strains, and the nucleotide sequences were submitted to the EMBL nucleotide sequence database (Table 2). The nucleic acid subsequences such as the -10 signal of a promoter, the RBS, the signal peptide of 81 bp and the hairpin structure were also present and were conserved among all strains tested (data not shown). As already indicated in Table 2, the nucleotide sequences of the gene porM1 differed among different strains of $M$. fortuitum.

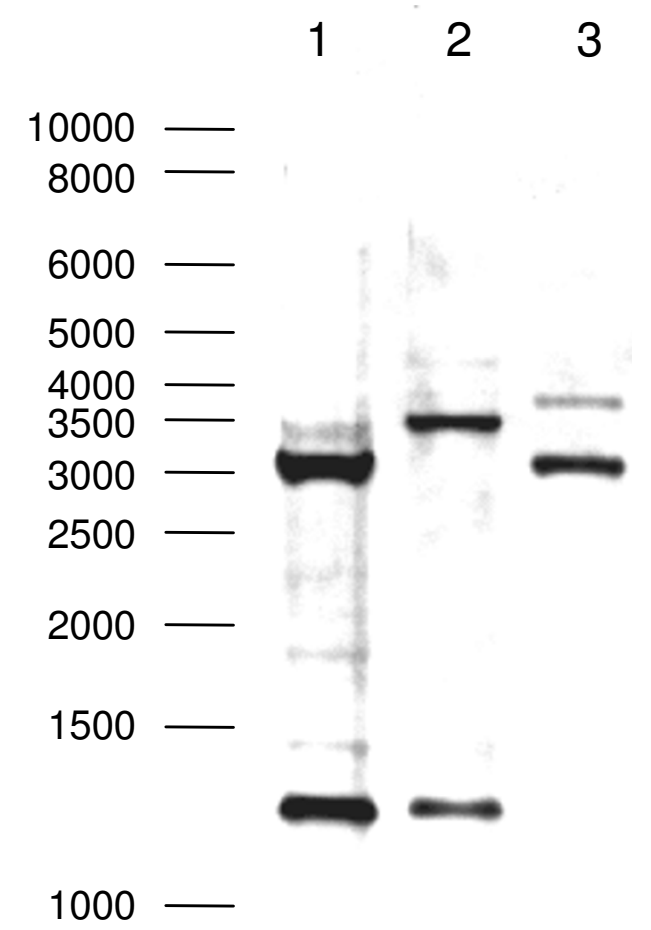

Figure 3

Occurrence of porin genes in $\mathbf{M}$. fortuitum. Chromosomal DNA of different strains was digested with Sacll and analysed by Southern Blotting using a probe derived from the porMI sequence. Lane I: M. fortuitum I085I/03; lane 2: $M$. fortuitum 10860/03; lane 3: M. fortuitum DSM 4662I.
The amino acid sequences of PorM1 among the $M$. fortuitum strains 10851/03 and 10860/03 including the type strain were identical (Figure 4 ). The mature PorM1 from M. fortuitum featured six amino acid substitutions compared to MspA.

Since the southern blot experiments had indicated the existence of two genes orthologous to $m s p A$ in $M$. fortuitum, we also attempted to clone and characterise the second porin gene. This porin gene, termed porM2, was amplified by PCR and cloned as a 918 bp fragment into the mycobacterial vectors pMV306 and pMV261, as described in the section Methods. The corresponding recombinant plasmids were named pSRa104 and pSRb103, respectively. Positive clones were confirmed by sequencing. As shown in Figure 2B, the insert of the plasmids contained an ORF of $648 \mathrm{bp}$, which turned out to be paralogous to the gene porM1. The $648 \mathrm{bp}$ ORF encodes a protein of 215 amino acids with an N-terminal signal sequence of 31 amino acids, which was predicted using the SignalP 3.0 Server at http://www.cbs.dtu.dk/services/ SignalP/[11]. The in silico analysis of the mature PorM2 showed a calculated molecular weight of the monomer of 19374 Da and a pI of 4.31, which were very similar to the calculated values of PorM1. A hypothetical -10 promoter sequence and a hypothetical RBS were located upstream of porM2. A hypothetical terminator sequence was, however, not detected (Figure 2B). The similarity between porM1 and porM2 from strains M. fortuitum 10851/03 and $10860 / 03$ on nucleotide level amounted to $94.1 \%$ and $95.3 \%$, respectively. The $m s p A$ gene revealed to be more similar to porM1 ( $87.4 \%$ to $88.4 \%$ similarity) than to porM2 (86.5\% similarity). Sequence comparison revealed that porM2 encodes a protein mainly differing from porM1 within the signal sequence. PorM2 from M. fortuitum $10851 / 03$ and 10860/03 exhibits an insertion of four amino acids and additional six amino acid exchanges within the signal peptide compared to PorM1 (Figure 4). Only one amino acid is replaced in the mature polypeptide [proline ${ }^{165}$ (PorM1) with alanine ${ }^{169}$ (PorM2)]. We sequenced a 1697 bp region comprising porM2, $500 \mathrm{bp}$ of its upstream region as well as 549 bp downstream of porM2. The DNA sequences flanking the porM1 and porM2 genes revealed no similarity with exception of the $13 \mathrm{bp}$ preceding the por $M$-coding sequences, which were identical in both regions. The 5' terminus of an ORF orthologous to a glycosyl transferase gene from $M$. tuberculosis CDC1551 (accession no.: AAK 48256) was detected upstream from porM2. An ORF orthologous to the gene for a pyridoxamine 5'-phosphate oxidase-related protein from M. vanbaalenii (accession no.: ZO 01208463) was present in the downstream region of porM2 (Figure 2B).

Using the primer pairs porM2-fw-hind and porM2-bwhpa or porM2-rna-fw and porM2-rna-bw (Table 1), porM2 

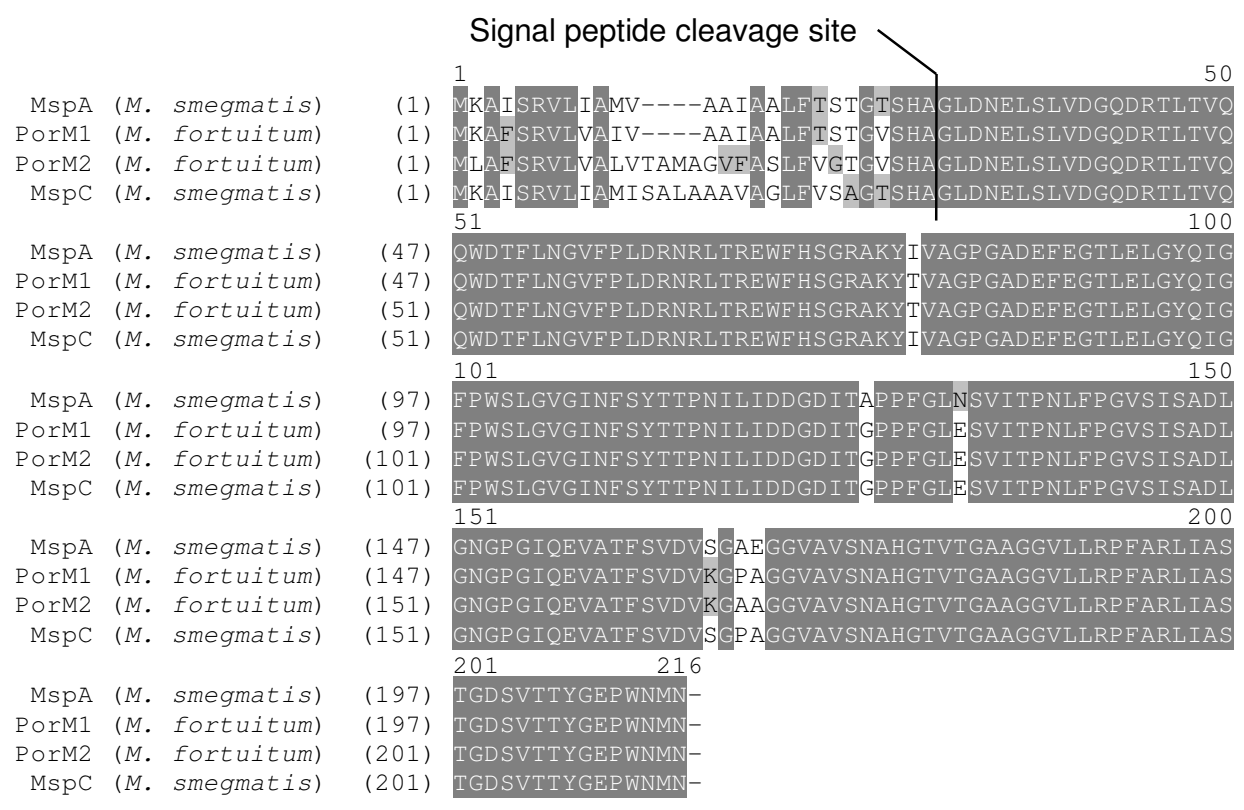

\section{Figure 4}

Alignment of PorMI and PorM2 from M. fortuitum and MspA and MspC from M. smegmatis. The start codon ATG and the stop codon TGA were chosen according to the sequence of $m s p$. The cleavage recognition site of the signal peptidase was predicted for PorMI, PorM2 and MspC using the SignalP 3.0 Server at http://www.cbs.dtu.dk/services/SignalP/[I I]. The predicted signal peptide cleavage sites corresponded to the signal peptide cleavage site of MspA [6]. Identical amino acids are dark grey, similar amino acids are light grey and different amino acids are not shaded. For PorMI and MspA an identity index of $94.8 \%$ was calculated, while PorM2 showed an amino acid identity of $90.7 \%$ to MspA.

was also detected in other strains analysed. No product was obtained using the plasmid pSSp107 carrying porM1 as template, demonstrating the specificity of this PCR approach for porM2.

\section{M. fortuitum strains express less porin compared to $M$. smegmatis}

The expression of the porins PorM1 and PorM2 were examined by 2D-Electrophoresis, Western Blotting, ELISA and qRT-PCR. For porin protein analysis, M. fortuitum pellets were lysed in POP05 (PBS 0.5\% (w/v) n-octylpolyoxyethylene $/ 0.2 \%$ EDTA) that was shown to selectively extract MspA [12]. For enhanced resolution and characterisation of the proteins, porin preparations were subjected to 2D-Electrophoresis. As shown in Figure 5A, about 50 protein spots were detected on the 2D-gel in M. fortuitum POP05 cell extracts. Western blot experiments with identical gels showed only one defined spot detected by the antiserum pAK MspA\#813 [6] (see Additional file 2). The protein had an apparent molecular mass of approximately $120 \mathrm{kDa}$, the expected size of the oligomeric porin, and an apparent pI of about 4, which corresponded well to the predicted pI of the mature protein of 4.31. Oligomers of the porin were readily detected in cell extracts of all M. for- tuitum strains as well as in extracts from M. smegmatis that served as a positive control. After extended exposition times, the monomer of the porin was also detected on Western Blots (data not shown). The Western Blots showed considerable differences in porin protein expression among the analysed strains (see Additional file 3). Additionally, ELISA experiments with POP05 extracts were performed to quantify the amount of porin in different strains. Different dilutions of cell extracts from the various strains were loaded into the wells of a microtiter plate and porins were detected using the polyclonal antibody pAK MspA\#813. Since M. bovis BCG does not possess orthologous porins [6], extracts of $M$. bovis BCG were employed as a control to detect the background. Amounts higher than $5 \mu \mathrm{g}$ per well turned out to be inapplicable due to saturation effects, and the detection of porin in cell extracts failed at concentrations of about $0.04 \mu \mathrm{g}$ per well. Therefore, the most eligible working range turned out to be $1 \mu \mathrm{g}$ of cell extract per well. Indeed, the amount of porin differed in various strains. The highest amount of porin was detected in the internal control M. smegmatis SMR5. The type strain M. fortuitum DSM 46621 exhibited porin amounts close to those of $M$. smegmatis, whereas the other two strains showed clearly decreased porin amounts 

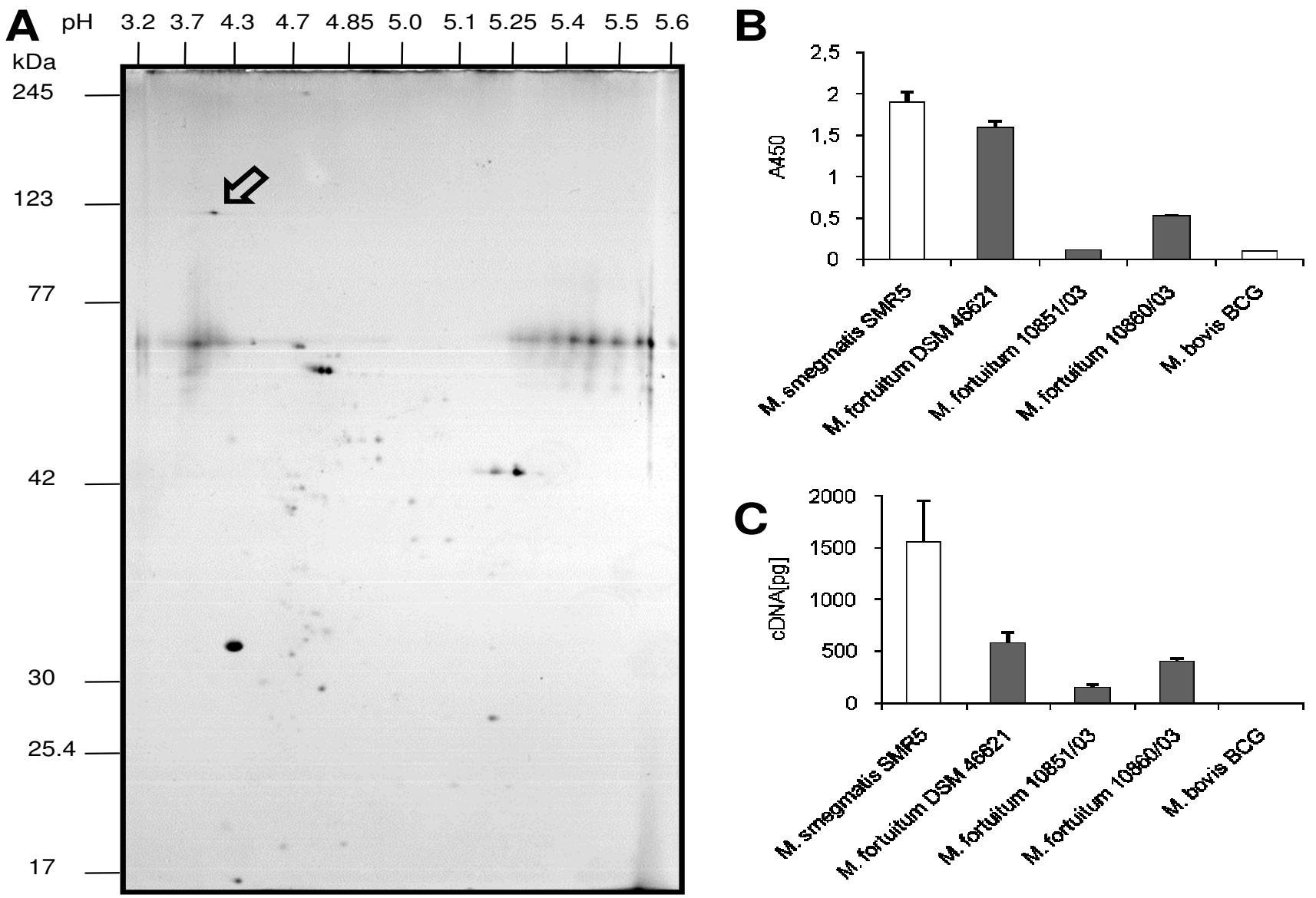

\section{Figure 5}

Detection of PorMs in M. fortuitum and M. smegmatis. 2D-Electrophoresis, Western Blot, ELISA and qRT-PCR experiments proved PorMs to be expressed in the analysed strains. Section A shows 2D-Electrophoresis of protein isolation from the strain M. fortuitum 10860/03 using the detergent nOPOE. The arrow indicates the porin spot proven by Western Blot analysis (see Additional file 2). Section B and C show comparative analysis of porin expression among RGM. Expression of porin was detected by means of ELISA (B) and qRT-PCR (C). Each value represents the mean ( \pm SD) of at least three independent experiments. B: Quantification of porin by means of ELISA in cell extracts of different mycobacteria using the polyclonal antibody pAk MspA\#8I3. C: RT-Real-time-PCR quantification of porin mRNA in various RGM using specific primers and probes for $m s p A$ or porM, respectively.

(Figure 5B). Notably, M. fortuitum 10851/03 exhibited the lowest amount of porin very close to the background, which was represented by the control M. bovis BCG.

Comparative expression analysis was also performed by means of quantitative reverse transcription polymerase chain reaction (qRT-PCR) using sequence-specific primers and probes (Table 1). The values were compared to porin expression in M. smegmatis. Because of the high degree of sequence conservation of the two paralogs porM1 and porM2, a qRT-PCR approach was established using primers and a dually labelled probe that hybridised to a region where both genes are identical (porM1 amplicon: nucleotide 132-232 and porM2 amplicon nucleotide 144-244, see also Table 1). This PCR approach enabled the quantification of the overall expression of the paralogs. As was already proven by the ELISA results, the highest porin mRNA expression was measured in $M$. smegmatis. It showed transcription rates about twice as high as the highest level among the M. fortuitum strains, which was detected in the type strain M. fortuitum DSM 46621. M. fortuitum 10851/03 exhibited the lowest transcription rate among all M. fortuitum strains (Figure 5C). The quantification of porM mRNA as well as the protein isolated from the various strains demonstrated consistence of transcriptional and translational levels and underlined the differential porin expression among the analysed $M$. fortuitum strains. 
MspA was shown to be accessible on the cell surface of $M$. smegmatis by using pAK MspA\#813 [8]. Since the expression analysis showed a differing amount of porin in $M$. fortuitum strains, M. fortuitum DSM 46621 and M. fortuitum 10860/03 (the strains with the highest porin expressions) were employed for detection of porins at the surface of intact mycobacteria. Porins were accessible at the surface of intact cells of M. fortuitum and were detected by the porin-specific antibody. Significantly higher absorption at $450 \mathrm{~nm}(\mathrm{P}<0.001)$ was measured for $M$. fortuitum DSM 46621 as well as M. fortuitum 10860/03 when compared to the relative backgrounds (see Additional file 4).

\section{PorM expression in the porin-deficient mutant strain $M$.} smegmatis $M L I O$ leads to improved growth

Heterologous expression of porM1 as well as porM2 was performed in the porin mutant strain M. smegmatis ML10 $(\triangle m s p A ; \Delta m s p C)$ to prove the functionality of encoded porins. For this purpose, the plasmids pSRa102 and pSRa104 harbouring porM1 and porM2, respectively, were introduced to M. smegmatis ML10. The plasmid pSSa100 [13] containing $m s p A$ was employed as a positive control.

First, the growth on Mycobacteria agar plates was quantified during four days after electroporation. The growth was compared to a strain harbouring only the vector pMV306. As it is shown in Figure 6A to 6D, heterologous expression of $m s p A$, por $M 1$ and por $M 2$ led to enhanced growth of complemented strains compared to the control. Figure 6A and 6B illustrate the growth retardation of strain M. smegmatis ML10 (pMV306) compared to the mspAcomplemented strain M. smegmatis ML10 (pSSa100). The growth of $M$. smegmatis ML10 was ameliorated by both, plasmid pSRa102 as well as plasmid pSRa104 (Figure 6C and 6D), although the complementation of the growth defect by these plasmids was less pronounced than by $m s p A$ expression using pSSa100. Heterologous expression of porM1 in the M. smegmatis mutant (Figure 6C) resulted in better growth than heterologous expression of porM2 (Figure 6D).

The quantification of growth rates of the strains by cfucounting confirmed these conclusions (Figure 6E). The strain complemented with $m s p A$ (containing pSSa100) had reached its final number of colonies after 72 hours. The transformant complemented with porM1 (containing pSRa102) also showed visible colonies after 72 hours, it had, however, not yet reached its final number of colonies after this time period. The strains containing pSRa104 (carrying porM2) and the empty vector pMV306 both showed visible colonies not until 96 hours, but ML10 (pSRa104) outnumbered ML10 (pMV306).
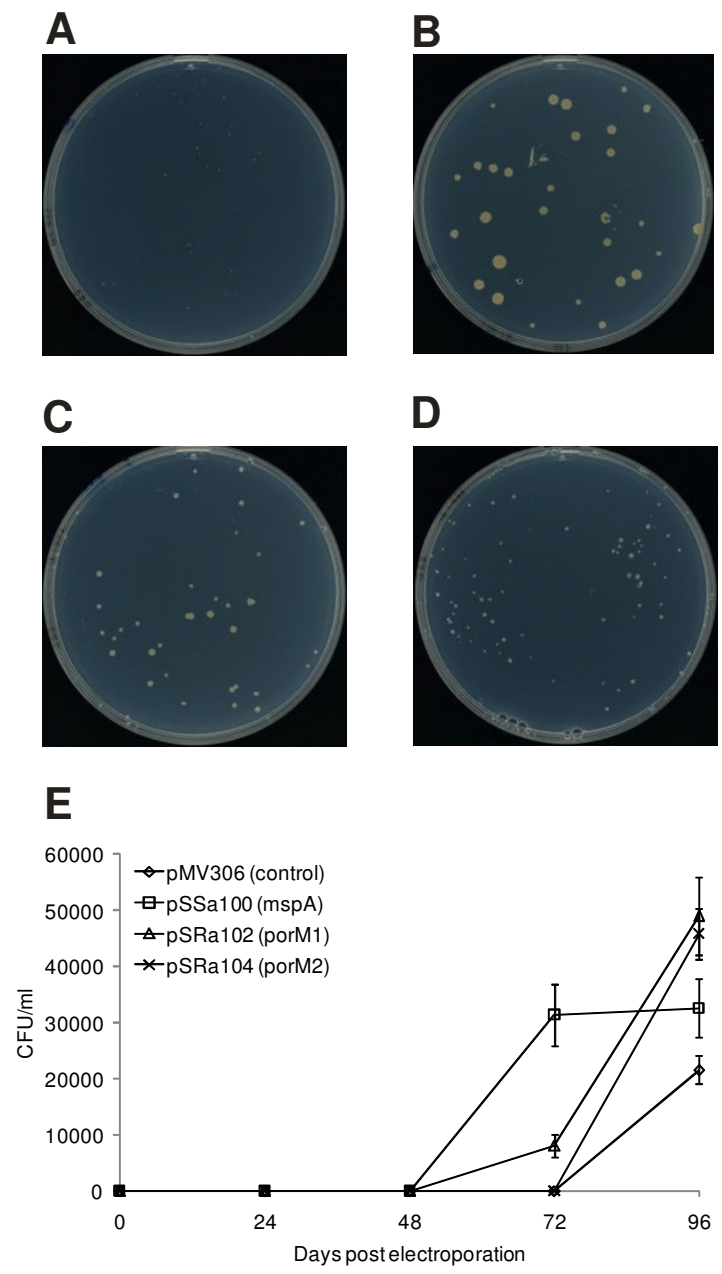

\section{Figure 6}

Complementation of the porin-deficient mutant strain $M$. smegmatis MLI 0 with porMI and porM2. M. smegmatis MLIO was transformed with the control vector pMV306 (A), the mspA-containing plasmid pSSa 100 (B), the porMl-containing plasmid pSRa 102 (C) and the porM2-containing plasmid pSRa 04 (D). After electroporation of the plasmids, dilutions of the transformed bacteria were plated onto Mycobacteria 7HI I agar with $25 \mu \mathrm{g} \mathrm{ml} \mathrm{m}^{-1}$ kanamycin and incubated for four days. Panel $(E)$ illustrates the result of an independent experiment showing the time course of the appearance of the colonies on Mycobacteria 7HII agar with $25 \mu \mathrm{g} \mathrm{ml} \mathrm{m}^{-1}$ kanamycin during four days after plating of a I:I0 dilution of the electroporated cells.

\section{Knock-down of porM expression by RNA antisense technique as well as over-expression of porMI or porM2 affect the growth rate of $M$. fortuitum}

Knock-down of porin expression in M. fortuitum was performed by generating the plasmid pSRr106, which carries a por $M$ antisense fragment (see Figure $2 \mathrm{~A}$ ) under the control of the $h s p 60$ promoter. The employed antisense 
sequence was first tested for non-specific binding performing a blast search at http://blast.ncbi.nlm.nih.gov/ Blast.cgi. The analysis ensured that the antisense fragment specifically binds to $m s p A$ class porins, such as porMs and did not show any hits to other sequences deposited in the database.

The efficiency of down-regulation via RNA antisense technique was proven by means of SYBR Green qRT-PCR using strain 10860/03. As shown in Additional file 5, the knock-down strain carrying the plasmid pSRr106 showed about four times lower porin expression compared to the control strain harbouring the vector PSHKLx1.

In order to over-express por $M$ genes in $M$. fortuitum, the coding sequences of porM1 from strain $M$. fortuitum $10851 / 03$ and of porM2 from strain 10860/03 were inserted downstream of the $h s p 60$ promoter in the vector pMV261 to generate plasmids pSRb101 and pSRb103, respectively.
We first studied the impact of the modified porM expression rates on the growth of bacteria freshly transformed with plasmids pSRr106, pSRb101 and pSRb103 as well as with the empty vectors PSHKLx1 and pMV261, serving as negative controls. Strains transformed with pSHKLx1 or pSRr106 were either selected by adding kanamycin (100 $\left.\mu \mathrm{g} \mathrm{ml}^{-1}\right)$ or hygromycin $\left(100 \mu \mathrm{g} \mathrm{ml}^{-1}\right)$ to the agar, while transformants electroporated with pMV261, pSRb101 or pSRb103 were selected by addition of kanamycin (100 $\mu \mathrm{g}$ $\left.\mathrm{ml}^{-1}\right)$. The clearest results were obtained with strains 10851/03 and DSM 46621 and are displayed in Figure 7(A, B, C-E, F, G, H-K). Knock-down of porM expression in both strains resulted in considerable growth reduction (Figure 7A, B and 7F, G) substantiating an important role of porins for the growth of M. fortuitum. This was further supported by the growth pattern of the 10851/03 derivatives over-expressing porM1 or porM2 (Figure 7C-E). Compared to $10851 / 03$ containing the empty plasmid pMV261, both derivatives over-expressing porM genes brought about a slight increase in average colony size on plates containing $100 \mu \mathrm{g} \mathrm{ml}^{-1}$ kanamycin. This effect was knock-down
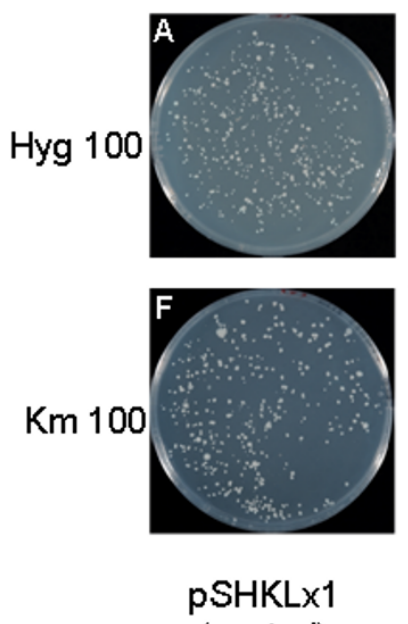
(control)
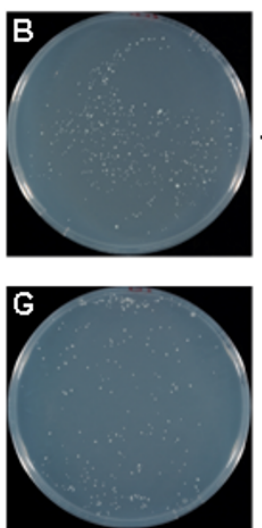

pSRr106 (knock-down)
$10851 / 03$
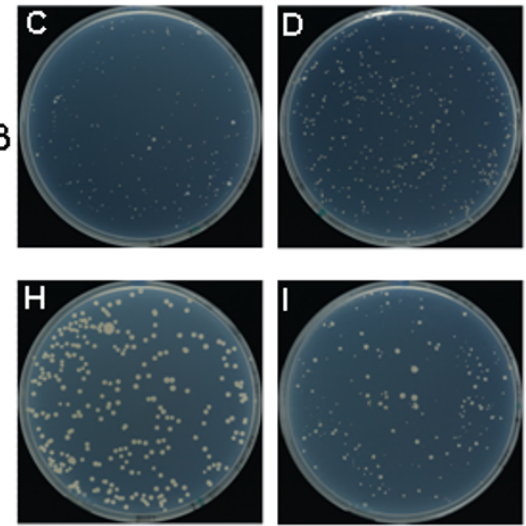

DSM 46621

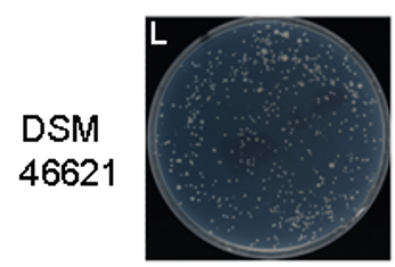

pMV261

(control)

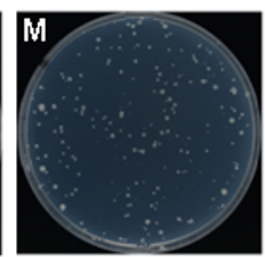

pSRb101

\section{over-expression}
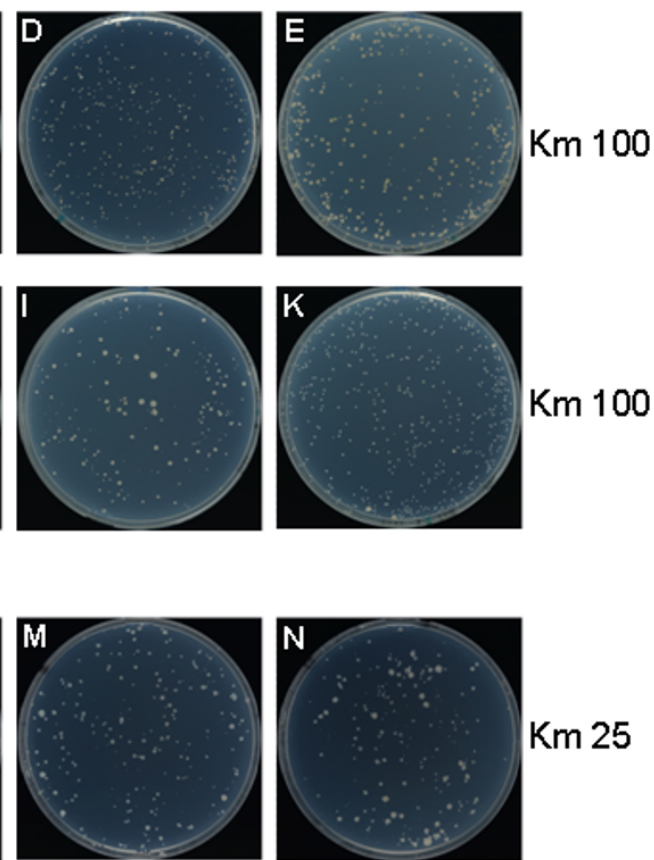

pSRb103

(+ porM2)

\section{Figure 7}

Effect of down-regulation and over-expression of porMI and porM2 on the growth of $M$. fortuitum. M. fortuitum strains I085I/03 (A-E) and DSM 4662I (F-N) were electoprorated with plasmids pSHKLxI (A, F), pSRr I06 (B, G), pMV26I (C,

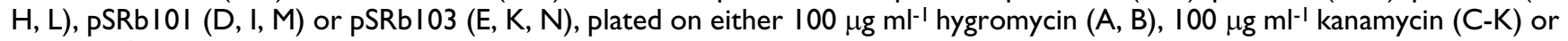
$25 \mu \mathrm{g} \mathrm{ml} \mathrm{m}^{-1}$ kanamycin (L-N) and incubated for four days. 
more pronounced in 10851/03 over-expressing porM2 than in the strain over-expressing porM1. In DSM 46621 the porin over-expression had an adverse effect on growth upon plating on $100 \mu \mathrm{g} \mathrm{ml}^{-1}$ kanamycin (Figure $7 \mathrm{H}-\mathrm{K}$ ). In order to figure out if this growth decrease was caused by an increased antibiotic uptake, we then plated the overexpressing DSM 46621 derivatives and the control on plates containing only $25 \mu \mathrm{g} \mathrm{ml}^{-1}$ kanamycin (Figure $7 \mathrm{~L}-$ $\mathrm{N})$. Under these conditions, the over-expression of porM genes slightly enhanced the growth. Again the increase in average colony size was more pronounced upon overexpression of porM2.

\section{Discussion}

In recent studies, our research has concentrated on the impact of the cell wall permeability on growth and intracellular persistence of mycobacteria. We were able to show that the porin pathway affects the intracellular persistence of different species in different ways. The findings suggest that intracellular persistence of mycobacteria depends, inter alia, on the balance between "walling-off" towards the hostile environment and the uptake of required compounds in the nutrient-depleted phagosomal environment $[5,13,14]$. To further examine this hypothesis, we are searching for more appropriate models. Different views have been expressed among scientists about whether $M$. smegmatis could serve as an appropriate model to study aspects related to virulence of highly pathogenic mycobacteria. A notable number of $M$. tuberculosis genes that are related to virulence but also play a housekeeping role share closely related orthologs in M. smegmatis. In the case of common mycobacterial genes, $M$. smegmatis was suggested as an appropriate model organism $[15,16]$. On the other hand, the physiological differences between $M$. smegmatis and $M$. tuberculosis were mentioned to narrow down the significance of direct comparisons [17]. Mutagenesis of porin genes in M. smegmatis allows the investigation of the impact of cell wall permeability on persistence. However, more appropriate models for such studies must naturally be able to survive and multiply intracellularly. Additionally, they must possess a known class of porin. These conditions are fulfilled by $M$. fortuitum, which was recently suggested as a model Mycobacterium [9]. This species is able to infect and grow in phagocytic cells [2,3] and also possesses porins orthologous to MspA. We therefore decided to identify and characterise porin genes from M. fortuitum.

The results of this study show that different strains including the type strain - of $M$. fortuitum possess orthologous porins of the MspA class. The amino acid sequences of PorM1 and PorM2 are highly conserved among the strains, whereas there is variability in their nucleotide sequence. PorM1 and PorM2 have the same apparent molecular mass as MspA and MspC, respectively. They are accessible at the surface of $M$. fortuitum. In detergent extracts of $M$. fortuitum mature oligomers of PorMs were detected, similar to M. smegmatis porin oligomers. As oligomer formation is necessary for channel activity [18], it can be concluded that $M$. fortuitum porins form functional pores in the OM. Mature PorM1 from M. fortuitum differs at only six amino acid positions from MspA. According to the studies of Faller et al. [7] and Mahfoud et al. [19] about the structure of MspA and its topology in the OM of $M$. smegmatis, it can be assumed that the amino acid replacements between PorM1 and MspA do not significantly affect the general porin structure. Remarkably, most of the exchanges are restricted to those residues, which are also variable within the Msp family. For example, the replacement of alanine ${ }^{138}$ with proline in the extracellular loop L9 between the $\beta$-sheets 9 and 10 supports the tight turn between the $\beta$-sheets and the change of direction. Interestingly, PorM2 does not feature the mentioned exchange of alanine with proline, which is the only amino acid exchange in the mature protein between PorM1 and PorM2. Faller et al. [7] proposed that the adjacent replacement of serine ${ }^{163}$ with lysine changes the antigenic properties of MspD compared to the other isomers. Although PorMs have the same exchange, they were readily detectable using a polyclonal antibody raised against MspA. The exchange of asparagine ${ }^{129}$ with glutamic acid within the periplasmatic loop L6 introduces a negative charge into the channel and may thus change the permeation properties slightly [7]. The replacement of isoleucine ${ }^{76}$ with threonine within the $\beta$-sheet $\beta 3$ should not affect the protein structure either, since both amino acids are C-beta branched amino acids and it is more favourable for them to lie between $\beta$-sheets [20].

The capacity of the encoded porins PorM1 and PorM2 to fulfil the function of a porin was tested in complementation experiments by introducing these genes into the double mutant strain M. smegmatis ML10 ( $\Delta m s p A ; \Delta m s p C)$ and observing the growth rate. Interestingly, porM1 had a stronger complementation effect than por $\mathrm{M} 2$, which was indicated by faster appearance of colonies and larger colony sizes on plates after electroporation. This may be explained by the higher similarity of porM1 to $m s p A$, which represents the main porin gene in M. smegmatis [8]. The antiserum raised against MspA binds well to PorMs, and the growth defect of the mutant strain $M$. smegmatis ML10 is reduced after complementation with porM1 and porM2. All mentioned features indicate similar functions and characteristics of the porins from M. smegmatis and $M$. fortuitum.

As mentioned above, mature PorM2 only differs from the mature PorM1 in one amino acid. More remarkable differences occur in the signal peptide of the two porins. The calculated cleavage site (SHA-GL) of the signal peptides of 
PorM1, PorM2, MspA and MspC is identical. However, the length of the signal peptides differs. While PorM1 and MspA have signal peptides composed of 27 amino acids, PorM2 and MspC possess extended signal peptides consisting of 31 amino acids. The length and primary structure of the signal peptide could be important for the transport and integration of the particular porin to the mycobacterial OM. It will be very informative to analyse the different regulation mechanisms determining the importance of the two genes for growth under different environmental conditions.

It was interesting that the expression of porM genes both at the transcriptional level and at the translational level consistently differed among the analysed strains as shown by the three employed approaches (Western Blot, ELISA and qRT-PCR). The results of both quantitative assays show the lowest porin expression among $M$. fortuitum strains in 10851/03 followed by 10860/03 and the type strain. The use of a polyclonal antibody, which recognises different epitopes of the protein and the consistency among the results of three different approaches allows drawing the conclusion that the porin expression in $M$. fortuitum is lower compared to M. smegmatis and also varies between the different strains. The high sequence conservation of the two paralogs PorM1 and PorM2 does not allow their expressions to be distinguished. Therefore, we consider the expression rates as overall values of both paralogs. As shown by qRT-PCR and ELISA, the porin expression in different strains of $M$. fortuitum was significantly lower than that of $M$. smegmatis. It was shown that $M$. smegmatis possesses $1000 \mathrm{MspA}$-like pores per $\mu \mathrm{m}^{2}$ cell wall [21]. Since the analysed strains of $M$. fortuitum exhibited a clearly lower por $M$ expression both at the transcriptional and the translational level, the amount of pores in the cell wall of $M$. fortuitum must be distinctly lower than 1000 pores per $\mu \mathrm{m}^{2}$ cell wall. According to our results, the amount of MspA-like pores in the analysed strains of $M$. fortuitum varies between 600 in M. fortuitum DSM 46621 and less than 100 per $\mu \mathrm{m}^{2}$ cell wall in M. fortuitum 10851/ 03, which exhibits the lowest amount of porin at all. It is interesting that the strain exhibiting the lowest porin expression is identical with the strain showing the slowest growth rate. This finding supports the hypothesis that porins play an important part in determining the generation time of mycobacteria.

To investigate the impact of the porins PorM1 and PorM2 on the growth rate of $M$. fortuitum, we generated strains over-expressing porM1 or porM2. Additionally, M. fortuitum knock-down strains were generated by antisense technique. This technique has contributed to the clarification of the function of many mycobacterial genes. Advantages are the possibility to analyse essential genes whose mutagenesis would be lethal and to repress genes present in several copies. Some examples of the application of the antisense technique in mycobacteria are the repression of ahpC from M. bovis [22], dnaA from M. smegmatis [23], FAP-P from M. avium subsp. paratuberculosis [24], pknF from M. tuberculosis [25] or MDP1 from $M$. bovis BCG [26]. A further advantage of knocking-down genes by antisense technique can be the possibility to repress paralogous genes in the same bacterium. As described in Dryselius et al. [27], the most effective region for antisense inhibition is the region covering the Shine-Dalgarno Sequence and the start codon. The authors furthermore show, that antisense RNAs of only 10-12 bases exhibit strong inhibitory effects on gene expression. The antisense fragment used in this study is identical to the corresponding region of porM1. While it displays a homology of $71.4 \%$ to porM2, the antisense fragment and porM2 still exhibit long stretches of identical nucleic acid sequences. Of particular importance is the similarity in the beginning of the antisense fragment covering the Shine-Dalgarno Sequence and the start codon (40 bp, 95\% identity). We therefore are convinced that a down-regulation of both, porM1 as well as porM2, may be achieved using the strategy described in this study. Deletion- or insertion mutagenesis of either porM1 or porM2 might result in complementation of the deleted porin gene by the remaining one. Such an effect has been observed in $M$. smegmatis, where the deletion of the mspA gene caused the activation of the transcription of $m s p B$ and/or $m s p D$ [28]. Mutagenesis of both porin genes in the same derivative, on the other hand, would probably restrain the diffusion across the $\mathrm{OM}$ to an extent compromising cellular functions.

The effects of an over-expression of porin in our $M$. fortuitum strains depended on characteristics of the strains as well as the amount of kanamycin added to the medium. The over-expression of porM1 and porM2 showed the most considerable influence on growth rate in strain 10851/03. Among the tested strains, 10851/03 has the slowest growth rate and produces least porin. Therefore, this strain probably benefits most from a better nutrient supply caused by porin over-production. Otherwise, the adverse effect of kanamycin on the growth rate was most pronounced in strain DSM 46621, which expresses the highest amount of porin among the analysed strains. Disposing of a relatively high amount of porin, this strain probably takes less advantage of an ameliorated nutrient supply and instead suffers most from more kanamycin diffusion into the cells. When the kanamycin concentration in the plates was reduced to $25 \mu \mathrm{g} \mathrm{ml}-1$, the overexpressing DSM 46621 derivatives did not show any growth inhibition compared to the control strain and even had a slight growth advantage. It seems that at this kanamycin concentration the beneficial effects of better nutrient influx slightly exceed the adverse effects of better 
antibiotic influx. The changes in growth behaviour in $10851 / 03$ as well as in DSM 46621 were more pronounced upon over-expression of porM2 compared to over-expression of porM1. The down-regulation of the expression of PorM1 together with PorM2 by antisensetechnology reduced the growth of both $M$. fortuitum strains to a similar and very low level suggesting that lack of porins in the knock-down strains strongly impairs the nutrient supply.

Our observations point to a passage of kanamycin through the PorM porins. Studies performed with $M$. smegmatis gave rise to contrarious conclusions [29,30]. Stephan et al. [29] observed no reduction of kanamycin resistance in a $m s p A$ mutant compared to the $M$. smegmatis wild type strain and Danilchanka and colleagues [30] had postulated using structural models that due to its size kanamycin cannot diffuse through the MspA porin. These differing results may in part be explained by the use of different experimental systems. Stephan et al. [29] employed a mutant strain, while we observed differential effects of kanamycin only in over-expressing strains. Furthermore, Stephan et al. [29] performed their studies with M. smegmatis and we observed strong strain-dependent variations even among different isolates within the same species. The amino acid exchanges occurring between MspA on the one hand and PorM1 and PorM2 on the other hand may be responsible for differences in channel properties of these porins and influence their permeability for kanamycin.

As we discussed earlier, the growth rate of mycobacteria may contribute to their pathogenicity [14]. Hence, it can be suggested that the low porin expression in $M$. fortuitum strains isolated from human patients compared to saprophytic species of RGM like M. smegmatis contributes to higher pathogenicity caused by an enhanced ability to multiply intracellularly. Interestingly, it was shown that the $m s p A$ expression in $M$. smegmatis is specifically downregulated at acidic $\mathrm{pH}[31]$. Moreover, the $M$. tuberculosis porin OmpATB, which belongs to the OmpA class of porins has been shown to be necessary for the persistence in the acidic milieu enabling $M$. tuberculosis to respond to reduced environmental $\mathrm{pH}[32,33]$. Although the MspA like porins do not belong to the OmpA class of porins, the results of these studies underline the role of porins concerning the intracellular persistence of mycobacteria.

An interesting result from various genome-sequencing projects of mycobacteria is that genome sizes of RGM and the pathogenic slow-growing mycobacteria largely differ. Highly pathogenic species like M. tuberculosis and M. leprae have genome sizes of about $4.4 \mathrm{Mb}$ and $3.27 \mathrm{Mb}$, respectively. On the other hand, M. smegmatis has a genome size of about $7 \mathrm{Mb}$, which is similar to that of the related actinomycete Streptomyces coelicolor. Brosch et al. [34] reviewed different data such as 16S rRNA sequences or genome sizes and suggested that the branch of slowgrowing mycobacteria represents the part of the genus that has evolved most recently. They proposed that the loss of genes rather than gain of genetic material by horizontal transfer contributed both to the pathogenicity of slowgrowing mycobacteria and to the fine-tuning of their virulence. Loss of efficient porins of the MspA class or a decreased density of porins in the OM plays an important role to "wall-off" toward the hostile phagosomal environment and thus is of particular importance for the evolution of a successful intracellular pathogen. The presence of several copies of porin genes and, in turn, a high density of efficient porins in the OM of $M$. smegmatis would provide a selective advantage for saprophytes. A decreased amount of efficient porins in the OM of pathogenic RGM like $M$. fortuitum may represent an evolutionary intermediate stage between saprophytic mycobacteria like $M$. smegmatis and the highly pathogenic slow-growing mycobacteria.

\section{Conclusion}

Our study provides detailed information about porin genes of the mspA class in M. fortuitum and their importance for the growth rate and susceptibility to antibiotics. Our future studies will concentrate on the elucidation of the role of PorM1 and PorM2 in survival and replication of phagocytosed M. fortuitum.

\section{Methods}

\section{Bacterial strains, cell lines and plasmids}

Mycobacterial strains (Table 3) were grown in Middlebrook 7H9 medium (BD Biosciences, Heidelberg, Germany), supplemented with $0.05 \%$ Tween 80 and either ADC (BD Biosciences) or DC ( 2 g glucose, $0.85 \mathrm{~g} \mathrm{NaCl}$, in $100 \mathrm{ml} \mathrm{H}{ }_{2} \mathrm{O}$ ) at $37^{\circ} \mathrm{C}$ without shaking, or on Mycobacteria $7 \mathrm{H} 11$ agar (BD Biosciences), supplemented with ADC (BD Biosciences). For selection of recombinant mycobacteria, media were supplemented when required with 25 to $100 \mu \mathrm{g} \mathrm{ml}^{-1}$ kanamycin or $100 \mu \mathrm{g} \mathrm{ml}^{-1}$ hygromycin B. E. coli DH5 $\alpha$ was grown in LB medium at $37^{\circ} \mathrm{C}$ [35]. Media were supplemented with $100 \mu \mathrm{g} \mathrm{ml}^{-1}$ kanamycin or 200 $\mu \mathrm{g} \mathrm{ml}^{-1}$ hygromycin B for selection of recombinant E. coli. All plasmids used in this study are described in Table 4.

\section{Measurement of growth rates in broth culture}

To compare the growth rates of $M$. fortuitum strains, Middlebrook 7H9/DC medium was inoculated with preparatory cultures to obtain an initial $\mathrm{OD}_{600}$ of 0.02 . During 16 days, the optical densities of the cultures were measured daily. Growth of the strains was monitored by quantification of the ATP content of the cultures with the luminescence-based kit BacTiter-Glo ${ }^{\mathrm{TM}}$ Microbial Cell Viability Assay (Promega). The luminescence was reported as rela- 
Table 3: Mycobacterial strains used in this work.

\begin{tabular}{lll}
\hline Strains & Characteristics & Reference \\
\hline M. smegmatis SMR5 & M. smegmatis $\mathrm{mc}^{2}$ I55 derivative, SMR & {$[42]$} \\
M. smegmatis MLI0 & SMR5 derivative, $\Delta m s p A$ and $\Delta m s p C$ & {$[4]$} \\
M. fortuitum DSM 4662I & Type strain; HYGR & This study \\
M. fortuitum 1085I/03 & Human patient isolate & This study \\
M. fortuitum I0860/03 & Human patient isolate; HYGR \\
M. bovis BCG Copenhagen & Vaccine strain & \\
\hline
\end{tabular}

HYG: hygromycin; SM: streptomycin

tive light units (RLU) with the microplate luminometer LB96V (EG\&G Berthold) [36].

\section{Molecular biology techniques and in silico analysis} Common molecular biology techniques were carried out according to standard protocols [35] or according to the recommendations of the manufacturers of kits and enzymes. Transformation of $E$. coli was performed according to the method of Hanahan [37]. PCR reactions were performed with the following kits: Taq DNA Polymerase (MBI Fermentas, St. Leon-Roth, Germany), BC Advantage GC Polymerase Mix (Takara Bio Europe S.A., Gennevilliers, France), BIO-X-ACT Short Mix and BIOTAQ DNA Polymerase (Bioline $\mathrm{GmbH}$, Luckenwalde, Germany). Sequencing reactions were performed by using the Prism Big Dye ${ }^{\mathrm{TM}}$ FS Terminator Cycle Sequencing Ready Reaction Kit from PE Applied Biosystems (Darmstadt, Germany).

Protein and nucleotide sequence analysis such as identification of DNA subsequences (e.g. promoters and terminators) was performed using the software packages MacVector $^{\mathrm{TM}}$ 7.2.3 (Accelrys, Cambridge, UK) and Lasergene (DNASTAR, Inc., Madison, WI, USA). Signal peptides were predicted using the SignalP 3.0 Server at http:// www.cbs.dtu.dk/services/SignalP/[11].

Phylogenetic relationships among the RGM were analysed using the program ClustalW in the MacVector ${ }^{\mathrm{TM}}$ 7.2.3 package. Before analysing the phylogenetic relationships, sequences were trimmed in order to start and finish at the same nucleotide position for all employed strains. Phylograms were obtained from nucleotide sequences using the neighbour-joining method with Kimura 2-Parameter distance correction [38].

\section{Cloning of porMl and porM2 from M. fortuitum and their detection in other strains of $M$. fortuitum}

In order to clone porin genes, genomic DNA from M. fortuitum was digested to completion with the restriction enzyme SacII and separated by agarose gel electrophoresis. The DNA was then transferred to the Hybond+ membrane (GE Healthcare, Munich, Germany) as described by Sambrook and Russell [35]. Porin genes were detected by means of Fluorescein-labelled probes using the primer pairs hpor and npor or mf-4IV-fw and mf-4-bw (Table 1) and the PCR Fluorescein Labelling Kit (Roche, Mannheim, Germany) according to the manufacturer's instructions. The region around $3000 \mathrm{bp}$ that was shown to hybridise to the probe was isolated out of the gel and was ligated into the unique SacII site of the plasmid pIV2 [39]. After transformation of E. coli $\mathrm{DH} 5 \alpha$, clones were screened by Dot Blot analysis. Inserts of two positive recombinant plasmids, pSSp107 and pSSp108, were sequenced. The inserts contained $m s p A$-related sequences referred to as porM1. Identification of orthologous genes among other members of $M$. fortuitum was performed by PCR using the primers komf-3f and komf-4b (Table 1), which were derived from the cloned genomic region of porM1.

For the cloning of porM2, genomic DNA from M. fortuitum 10851/03 DNA was digested with the restriction enzyme SmaI and a 4200 bp SmaI fragment that had shown to hybridise to the Fluorescein-labelled probe before was eluted from the agarose gel and ligated into the SmaI site of pLITMUS38 (New England Biolabs, Frankfurt, Germany) and clones were screened as mentioned above. The insert of the only positive clone was sequenced. A $181 \mathrm{bp}$ sequence similar to the $3^{\prime}$ terminus of the coding sequence of porM1 was identified, while the following 256 bp of the 3' flanking region showed no similarity to the porM1 flank. A PCR primer within the porM2 flanking region (porM2-51-bw) and another primer hybridising to the first 19 bp of the porM1 coding sequence (porM2-51$\mathrm{fw}$ ) were used to amplify porM2 sequences (Table 1). After sequencing the PCR product, a reverse PCR approach was adapted to discover the 5 ' flanking region of porM2 including its start. Therefore, genomic DNA of M. fortuitum 10851/03 was digested with NcoI. The DNA fragments were circularised by ligation. Then a PCR was performed using the reverse primers porM2-rev- 1 and porM2-rev-2 (Table 1) and the product was sequenced to obtain a complete sequence of por $\mathrm{M} 2$ and its flanking regions. The primers porM2-fw-hind (located 268 bp upstream of the porM2 coding sequence [CDS]) and porM2-bw-hpa (located directly downstream of the porM2 cds) (Table 1) were derived from the sequence mentioned and were chosen to amplify and clone porM2 
and its regulatory sequences. The 918 bp product was cloned into the HindIII/HpaI restriction sites of the integrative mycobacterial vector pMV306 [40] and the shuttle vector pMV261 [40] to generate the recombinant plasmids pSRa104 and pSRb103, respectively. Positive clones were verified by sequencing. PorM2 was detected in other strains using the primer pairs porM2-fw-hind and porM2bw-hpa or porM2-rna-fw and porM2-rna-bw (Table 1).

\section{Detection of porins by Western Blot and 2-D Electrophoresis}

M. smegmatis MspA as well as porins from M. fortuitum were extracted in PBS buffer supplemented with $0.5 \%$ (w/ v) n-octylpolyoxyethylene (nOPOE, Bachem, Heidelberg) and $0.2 \%$ EDTA (POP05), slightly modifying the method of Heinz and Niederweis [12]. Mycobacteria were grown to an $\mathrm{OD}_{600}$ of up to 1 . Subsequently, about $150 \mathrm{mg}$ of mycobacteria (wet weight) were washed twice in PBS buffer supplemented with 0.2\% EDTA. Pellets were resuspended in POP05 using a ratio of $200 \mu \mathrm{l}$ POP05 per 100 mg mycobacteria and were incubated at $100^{\circ} \mathrm{C}$ for 30 min. Afterwards, cell debris was sedimented by centrifugation at $27,000 \times \mathrm{g}$ and $4{ }^{\circ} \mathrm{C}$ and the supernatant was transferred to a new tube. Quantification of protein samples was carried out using the BCA Protein Assay Reagent Kit (Pierce, Rockford, IL, USA). Western Blot analysis was performed using the antiserum pAK MspA\#813 as described previously [13]. For 2D-analysis, about $75 \mu \mathrm{g}$ of protein was precipitated by acetone and pellets were washed with $70 \%$ acetone to desalt the sample. Afterwards pellets were resuspended in $200 \mu \mathrm{l}$ Rehydration solution (8 M Urea, 0.5\% CHAPS, $0.2 \%$ DTT, $0.5 \%$ Pharmalyte, $0.002 \%$ Bromphenol blue), incubated for $5 \mathrm{~h}$ at room temperature and loaded on IPG strips pH 3-5.6 NL, $11 \mathrm{~cm}$ (GE Healthcare). The strips were focused on an Ettan pIGphorII unit and the second dimension was run on vertical 10\% SDSPAGE gels using the Ettan Daltsix electrophoresis unit (GE Healthcare) according to the manufacturer's instructions. The gels were silver-stained using Roti-Black P (Carl Roth GmbH, Karlsruhe, Germany). The porin was detected by Western Blotting as mentioned above.

\section{Differential expression analysis of porins by $q R T-P C R$ and ELISA}

Expression of porin genes in the different strains was determined by means of qRT-PCR using the Mx3000P Real-time PCR System (Stratagene, La Jolla, CA, USA) or the StepOnePlus ${ }^{\mathrm{TM}}$ Real-Time PCR-System (Applied Biosystems). Mycobacteria were grown to an $\mathrm{OD}_{600}$ of 0.8 and RNA was extracted according to the method of Bashyam and Tyagi [41]. 1 or $5 \mu \mathrm{g}$ of the RNA was treated prior to qRT-PCR with RNase-Free DNase (Fermentas $\mathrm{GmbH}$, St. Leon-Roth, Germany). Reverse transcription of mycobacterial RNA was carried out using the RevertAid ${ }^{\mathrm{TM}}$ M-MuLV Reverse Transcriptase (Fermentas $\mathrm{GmbH}$ ) and hexamers or the Access RT-PCR System (Promega, Mannheim, Germany) according to the manufacturer's protocols. The porin cDNA from M. smegmatis SMR5 [42] and M. fortuitum was quantified either by amplifying a fragment of about 100 bp using the primers (mspATaqfw, mspATaqbw, mfpqPCRfw and mfpqPCRrev) as well as TaqMan-probes (mspATaqProbe and mfpqPCRprobe) or the primers porM1-51-sybr-fw and bw based on SYBR Green detection chemistry (Table 1 ). The qPCR reactions were performed using the SensiMix DNA Kit (Quantace Ltd., Berlin, Germany) or the Access RT-PCR System (Promega) according to the manufacturer's protocol. TaqMan quantification was carried out by running a first step at $95^{\circ} \mathrm{C}$ for $10 \mathrm{~min}$ followed by 40 cycles with $30 \mathrm{~s}$ at $95^{\circ} \mathrm{C}$ and $1 \mathrm{~min}$ at $58^{\circ} \mathrm{C}$. SYBR Green quantification was performed by initial $10 \mathrm{~min}$ at $95^{\circ} \mathrm{C}$ followed by 40 cycles with $15 \mathrm{~s}$ at $95^{\circ} \mathrm{C}, 10 \mathrm{~s}$ at $58^{\circ} \mathrm{C}$ and $20 \mathrm{~s}$ at $72^{\circ} \mathrm{C}$. Afterwards, the amplicon's melting temperature was determined ramping the temperature from $60^{\circ} \mathrm{C}$ to $90^{\circ} \mathrm{C}$ by $0.5^{\circ} \mathrm{C}$ steps and acquiring the fluorescence signal. cDNA amounts were determined by three measurements for each sample using a calibration curve established with known amounts of linearised pSSa100 [13] in case of $M$. smegmatis or pSSp107 in case of $M$. fortuitum. DNase treated and non-reverse-transcribed controls were performed with the same samples to guarantee the absence of contaminating genomic DNA.

In addition to the qRT-PCR experiments, the amount of porin in isolates of $M$. fortuitum and M. smegmatis was determined by Enzyme-Linked Immunosorbent Assay (ELISA). Protein was isolated from mycobacteria using the detergent nOPOE as described above. The isolated protein (15 $\mu \mathrm{l}$ corresponding approximately to $25 \mu \mathrm{g}$ ) was diluted in $50 \mathrm{mM} \mathrm{NaHCO}_{3}$, pH 9.6 to yield a protein concentration of $1 \mu \mathrm{g} / 100 \mu \mathrm{l}$. Aliquots $(100 \mu \mathrm{l})$ of the sample and dilutions thereof were loaded to wells of a Nunc-Immuno Maxisorp Module (Nalgene Nunc International, NY, USA). After incubating the samples at $4{ }^{\circ} \mathrm{C}$ overnight, wells were washed twice with TBS-T (50 mM Tris- $\mathrm{HCl}, \mathrm{pH}$ 7.8, $150 \mathrm{mM} \mathrm{NaCl}, 1 \mathrm{mM} \mathrm{MgCl}_{2}$ and $0.05 \%$ Tween 80 ). The surface was blocked with $3 \%$ powdered skim milk in TBS for $1.5 \mathrm{~h}$ at room temperature followed by three steps of washing with TBS-T. Samples were then treated with the primary antibody for $1.5 \mathrm{~h}$ at room temperature, using a 1:1500 dilution of the antiserum pAK MspA\#813 [8] in TBS. The wells were washed five times with TBS-T and were incubated for $1 \mathrm{~h}$ at room temperature with a 1:7500 dilution of Peroxidase-conjugated AffiniPure F (ab') 2 Fragment Goat Anti-Rabbit IgG $(\mathrm{H}+\mathrm{L})$ (Jackson Immuno Research, Soham, UK) in TBS. After five steps of washing, the reaction was performed using the SureBlue ${ }^{\mathrm{TM}} \mathrm{TMB}$ Microwell Peroxidase Substrate (KPL, Gaithersburg, MD, USA) according to the instructions of the manufacturer. 
Absorption at $450 \mathrm{~nm}$ was measured with the microplate reader SPECTRA Fluor (TECAN, Crailsheim, Germany).

\section{Detection of PorMs at the surface of mycobacteria by means of quantitative microwell immunoassays}

$40 \mathrm{ml}$ of mycobacterial culture was harvested at $\mathrm{OD}_{600}$ of 0.8, washed with PBS-T and the pellet was resuspended in $1 \mathrm{ml}$ PBS-T. $200 \mu \mathrm{l}$ aliquots were then incubated for 30 min on ice with $1 \mu \mathrm{l}$ of antiserum (pAK MspA\#813); for detection of background pre-immune serum was given to the samples. Afterwards $1 \mathrm{ml}$ PBS-T was given to each sample; mycobacteria were harvested by centrifugation and washed once with PBS-T. Pellets were resuspended in $100 \mu \mathrm{l}$ of PBS-T, $1 \mu \mathrm{l}$ of the secondary Peroxidase-conjugated AffiniPure F (ab') 2 Fragment Goat Anti-Rabbit IgG $(\mathrm{H}+\mathrm{L})$ (Jackson Immuno Research) was added to each sample and bacilli were incubated on ice for $30 \mathrm{~min}$. After addition of $1 \mathrm{ml}$ PBS-T, mycobacteria were pelleted by centrifugation and were washed once with PBS-T. Pellets were then resuspended in $500 \mu \mathrm{l}$ of PBS-T, and $100 \mu \mathrm{l}$ of dilutions thereof were transferred to wells of a NuncImmuno Polysorp Module (Nalgene Nunc International). After addition of $100 \mu \mathrm{l}$ SureBlue ${ }^{\mathrm{TM}} \mathrm{TMB}$ Microwell Perox- idase Substrate (KPL) and stopping the reaction by addition of $50 \mu \mathrm{l} 1 \mathrm{M} \mathrm{HCl}$, the reaction was detected by the reader SPECTRAFluor (TECAN).

\section{Complementation of the porin-deficient mutant strain $M$. smegmatis $M L I O$ with porMI and porM2}

The ability of porM1 and porM2 to complement the growth defect of M. smegmatis ML10 ( $\Delta m s p A ; \Delta m s p C)$ [4] was examined by electroporation with the plasmids pSRa102, pSRa104, pSSa100 (Table 4) as well as the control pMV306. $750 \mathrm{ng}$ of each plasmid was electroporated into M. smegmatis ML10 as described in Sharbati-Tehrani et al. [13]. After electroporation the cells were diluted and plated onto Mycobacteria 7H11 agar supplemented with kanamycin $(25 \mu \mathrm{g} / \mathrm{ml})$ for the assessment of growth after four days and for the quantification of growth by cfu counting during four days.

\section{Knock-down of porM expression and over-expression of porMl or porM2 in M. fortuitum}

In order to accomplish a simultanous knock-down of porM1 and porM2, we generated a plasmid containing a transcriptional fusion of the $h s p 60$ promoter with the 5

Table 4: Plasmids used in this work.

\begin{tabular}{|c|c|c|}
\hline Plasmids & Characteristics & Reference \\
\hline plV2 & $\begin{array}{l}\text { cloning vector with an origin of replication functional in Enterobacteriacea and a kanamycin resistance } \\
\text { gene }\end{array}$ & [39] \\
\hline pLitmus38 & $\begin{array}{l}\text { cloning vector with the origin of replication from pUC, an ampicillin resitance gene and the lac } Z \text { ' gene } \\
\text { for blue/white selection }\end{array}$ & New England Biolabs \\
\hline pMV306 & $\begin{array}{l}\text { cloning vector replicating in } E \text {. coli with the kanamycin resistance gene aph from transposon Tn } 903 \text { and } \\
\text { the gene for the integrase and the attP site of phage L5 for integration into the mycobacterial genome }\end{array}$ & {$[40]$} \\
\hline pMV26I & $\begin{array}{l}\text { Mycobacterium/E. coli shuttle vector with the kanamycin resistance gene aph from transposon } \mathrm{Tn} 903 \text { and } \\
\text { the promoter from the } h s p 60 \text { gene from M. tuberculosis }\end{array}$ & {$[40]$} \\
\hline pSHKLxl & $\begin{array}{l}\text { Mycobacterium/E. coli shuttle vector with a kanamycin resistance gene from } \mathrm{Tn} 5 \text {, a hygromycin } \\
\text { resistance gene and the promoter from the } h s p 60 \text { gene from } M \text {. tuberculosis }\end{array}$ & {$[43]$} \\
\hline pSSal00 & pMV306 with a 3429 bp genomic DNA fragment from M. smegmatis SMR5 carrying mspA & {$[13]$} \\
\hline pSSp 107, pSSp 108 & pIV2 with a 2895 bp genomic DNA fragment from M. fortuitum 10860/03 carrying the porMI gene & This study \\
\hline pSRbIOI & pMV26I carrying the porMI gene from M. fortuitum I0860/03 & This study \\
\hline pSRbI03 & pMV26I carrying the porM2 gene from M. fortuitum I085I/03 & This study \\
\hline pSRal02 & pMV306 carrying the porMI gene from M. fortuitum I0860/03 & This study \\
\hline pSRal04 & pMV306 carrying the porM2 gene from M. fortuitum I085I/03 & This study \\
\hline pSRrI06 & $\begin{array}{l}\text { PSHKLxI carrying a } 100 \text { bp genomic DNA fragment from M. fortuitum 10860/03 containing the } \\
\text { beginning of the porMI gene with the SD-sequence in antisense-orientation with respect to the hsp } 60 \\
\text { promoter }\end{array}$ & This study \\
\hline
\end{tabular}


region of porM genes. The primers porM1-as-1 and porM1-as-2 were used to amplify a 100 bp PCR amplicon covering the 5' region of porM1 including the Shine-Dalgarno Sequence. The PCR product was cloned into the BamHI site of pSHKLx1 [43], and recombinant plasmids containing the insert in antisense orientation with respect to the $h s p 60$ promoter were identified by sequencing. Afterwards, the selected recombinant plasmid pSRr106 was introduced into $M$. fortuitum by electroporation. The knock-down efficiency of the introduced antisense RNA was analysed at transcriptional level. For this purpose, RNA was isolated from $M$. fortuitum strains containing either pSRr106 or pSHKLx1, and porin expression was measured by SYBR Green qRT-PCR as described above.

Over-expression of porM1 or porM2, was achieved by introducing plasmids pSRb101 or pSRb103, respectively, into M. fortuitum.

\section{Authors' contributions}

SS conceived the study, performed experiments and analyses and wrote and edited the manuscript. KS performed experiments, supervised the work of SR, HW and RA and designed their experiments. SR, HW, RA, VT and RK performed experiments and analyses. AL contributed to the experimental designs, writing and composition of the manuscript. All authors read and approved the final manuscript.

\section{Additional material}

\section{Additional file 1}

Growth rate of the $\mathrm{M}$. fortuitum strains 10851/03, 10860/03 and DSM 46621. Logarithmic display of the growth curves shown in Figure 1. The growth rate of the strains was measured by quantification of the ATP-content [displayed as relative light units (RLU)] in broth cultures. Click here for file

[http://www.biomedcentral.com/content/supplementary/14712180-9-31-S1.ppt]

\section{Additional file 2}

Detection of the PorM spot on the 2D-PAGE by Western Blot analysis. Detection was performed using the porin-specific antiserum $p A K$ MspA\#813 on the blotted 2D-PAGE shown in Figure 5A. Only one protein spot was identified possessing an apparent molecular mass of approximately $120 \mathrm{kDa}$ and an apparent pI of about 4 . The arrow indicates the identified spot.

Click here for file

[http://www.biomedcentral.com/content/supplementary/14712180-9-31-S2.ppt]

\section{Additional file 3}

Western Blot analysis of PorMs in M. fortuitum. Porin expression in members of the $\mathrm{M}$. fortuitum-group was studied by Western blotting. 10$30 \mu \mathrm{g}$ of protein extracted with nOPOE was separated by 1D-SDS-PAGE and detected by the antiserum $P A K M s p A \# 813$. Lanes 1-4: 1, M. smegmatis SMR5 (10 $\mu \mathrm{g}) ; 2$, M. fortuitum DSM 466211 (30 $\mu \mathrm{g}) ; 3, \mathrm{M}$. fortuitum 10851/03 (30 $\mu \mathrm{g}) ; 4$, M. fortuitum 10860/03 (30 $\mu \mathrm{g}$ ). Click here for file

[http://www.biomedcentral.com/content/supplementary/14712180-9-31-S3.ppt]

\section{Additional file 4}

Detection of PorMs on the surface of $\mathrm{M}$. fortuitum. Detection was performed using the porin-specific antiserum $p A K M s p A \# 813$ in quantitative microwell immunoassays. Each column represents the mean $( \pm S D)$ of 8 measurements. Asterisks indicate significant differences between the samples, which were treated with $P A K M s p A \# 813$ and backgrounds according to the paired Student's $t$-test $(P<0.001)$.

Click here for file

[http://www.biomedcentral.com/content/supplementary/1471-

2180-9-31-S4.ppt]

\section{Additional file 5}

Knock-down of porins in M. fortuitum 10860/03 by means of antisense technology using the plasmid pSRr106. The amount of porM1/ porM2 mRNA was quantified by means of qRT-PCR and was normalised with 16S rRNA. Compared to the reference strain M. fortuitum 10860/ 03 ( $p S H K L x 1$ ) the amount of porM mRNA in the down-regulated strain 10860/03 (pSRr106) was reduced by about $75 \%$.

Click here for file

[http://www.biomedcentral.com/content/supplementary/14712180-9-31-S5.ppt]

\section{Acknowledgements}

We would like to thank Prof. Dr. Michael Niederweis (University of Alabama, Birmingham, $A L$ ) for providing the antiserum and the $M$. smegmatis strain MLI0. We also thank Dr. Rüsch-Gerdes (Nationales Referenzzentrum für Mykobakterien, Borstel) for providing the $M$. fortuitum strains 10851/03 and 10860/03. Furthermore, we thank Prof. Dr. Robertson (Imperial College, London) and Prof. Dr. Jacobs (Howard Hughes Medical Institute, New York) for providing plasmids PSHKLxI and pMV26I, respectively. We are grateful to Elisabeth Kamal for excellent technical assistance. Kira Schramm was supported by a European Union Equal Project grant.

\section{References}

I. Brown-Elliott BA, Wallace RJ Jr: Clinical and taxonomic status of pathogenic nonpigmented or late-pigmenting rapidly growing mycobacteria. Clin Microbiol Rev 2002, I 5:71 6-746.

2. Cirillo JD, Falkow S, Tompkins LS, Bermudez LE: Interaction of Mycobacterium avium with environmental amoebae enhances virulence. Infect Immun 1997, 65:3759-3767.

3. Da Silva TR, De Freitas JR, Silva QC, Figueira CP, Roxo E, Leao SC, De Freitas LA, Veras PS: Virulent Mycobacterium fortuitum restricts NO production by a gamma interferon-activated J774 cell line and phagosome-lysosome fusion. Infect Immun 2002, 70:5628-5634.

4. Stephan J, Stemmer V, Niederweis M: Consecutive gene deletions in Mycobacterium smegmatis using the yeast FLP recombinase. Gene 2004, 343:181-190.

5. Sharbati-Tehrani S, Stephan J, Holland G, Appel B, Niederweis M, Lewin A: Porins limit the intracellular persistence of Mycobacterium smegmatis. Microbiology 2005, I 5 I :2403-24I0. 
6. Niederweis M, Ehrt S, Heinz C, Klocker U, Karosi S, Swiderek KM, Riley LW, Benz R: Cloning of the mspA gene encoding a porin from Mycobacterium smegmatis. Mol Microbiol 1999, 33:933-945.

7. Faller M, Niederweis M, Schulz GE: The structure of a mycobacterial outer-membrane channel. Science 2004, 303:I I89-I I 92.

8. Stahl C, Kubetzko S, Kaps I, Seeber S, Engelhardt H, Niederweis M: MspA provides the main hydrophilic pathway through the cell wall of Mycobacterium smegmatis. Mol Microbiol 200I, 40:45I-464.

9. Philips JA, Rubin EJ, Perrimon N: Drosophila RNAi screen reveals CD36 family member required for mycobacterial infection. Science 2005, 309: I25I-I253.

10. Adekambi T, Drancourt M: Dissection of phylogenetic relationships among 19 rapidly growing Mycobacterium species by I6S rRNA, hsp65, sodA, recA and rpoB gene sequencing. Int j Syst Evol Microbiol 2004, 54:2095-2 105 .

II. Bendtsen JD, Nielsen H, von Heijne G, Brunak S: Improved prediction of signal peptides: SignalP 3.0. J Mol Biol 2004, 340:783-795.

12. Heinz C, Niederweis M: Selective extraction and purification of a mycobacterial outer membrane protein. Analytical biochemistry 2000, 285: II3-I20.

13. Sharbati-Tehrani S, Meister B, Appel B, Lewin A: The porin MspA from Mycobacterium smegmatis improves growth of Mycobacterium bovis BCG. Int J Med Microbiol 2004, 294:235-245.

14. Lewin A, Sharbati-Tehrani S: [Slow growth rate of mycobacteria. Possible reasons and significance for their pathogenicity]. Bundesgesundheitsblatt Gesundheitsforschung Gesundheitsschutz 2005 , 48: $1390-1399$

15. Reyrat JM, Kahn D: Mycobacterium smegmatis: an absurd model for tuberculosis? Trends Microbiol 200I, 9:472-474.

16. Tyagi JS, Sharma D: Mycobacterium smegmatis and tuberculosis. Trends Microbiol 2002, 10:68-69.

17. Barry CEl: Mycobacterium smegmatis: an absurd model for tuberculosis? Response from Barry, III. Trends Microbiol 200I, 9:473-474.

18. Niederweis M: Mycobacterial porins - new channel proteins in unique outer membranes. Mol Microbiol 2003, 49: I I67-I I 77.

19. Mahfoud M, Sukumaran S, Hulsmann P, Grieger K, Niederweis M: Topology of the porin MspA in the outer membrane of Mycobacterium smegmatis. J Biol Chem 2006, 281:5908-59I5.

20. Betts MJ, Russell RB: Amino acid properties and consequences of subsitutions. In Bioinformatics for Geneticists Edited by: Barnes MR, Gray IC. West Sussex, United Kingdom: Wiley and Sons; 2003:289-3I6

21. Engelhardt $H$, Heinz $C$, Niederweis M: A tetrameric porin limits the cell wall permeability of Mycobacterium smegmatis. Biol Chem 2002, 277:37567-37572.

22. Wilson T, de Lisle GW, Marcinkeviciene JA, Blanchard JS, Collins DM: Antisense RNA to ahpC, an oxidative stress defence gene involved in isoniazid resistance, indicates that AhpC of Mycobacterium bovis has virulence properties. Microbiology 1998, 144:2687-2695

23. Greendyke R, Rajagopalan M, Parish T, Madiraju MV: Conditional expression of Mycobacterium smegmatis dnaA, an essential DNA replication gene. Microbiology 2002, 148:3887-3900.

24. Secott TE, Lin TL, Wu CC: Mycobacterium avium subsp. paratuberculosis fibronectin attachment protein facilitates M-cell targeting and invasion through a fibronectin bridge with host integrins. Infect Immun 2004, 72:3724-3732.

25. Deol P, Vohra R, Saini AK, Singh A, Chandra H, Chopra P, Das TK, Tyagi AK, Singh Y: Role of Mycobacterium tuberculosis Ser/Thr kinase PknF: implications in glucose transport and cell division. J Bacteriol 2005, 187:34|5-3420.

26. Lewin A, Baus D, Kamal E, Bon F, Kunisch R, Maurischat S, Adonopoulou M, Eich K: The mycobacterial DNA-binding protein (MDPI) from Mycobacterium bovis BCG influences various growth characteristics. BMC Microbiol 2008, 8:91.

27. Dryselius R, Aswasti SK, Rajarao GK, Nielsen PE, Good L: The translation start codon region is sensitive to antisense PNA inhibition in Escherichia coli. Oligonucleotides 2003, 13:427-433.

28. Stephan J, Bender J, Wolschendorf F, Hoffmann C, Roth E, Mailander $\mathrm{C}$, Engelhardt H, Niederweis M: The growth rate of Mycobacterium smegmatis depends on sufficient porin-mediated influx of nutrients. Mol Microbiol 2005, 58:7|4-730.
29. Stephan J, Mailaender C, Etienne G, Daffé M, Niederweis M: Multidrug resistance of a porin deletion mutant of Mycobacterium smegmatis. Antimicrob Agents Chemother 2004, 48:4163-4170.

30. Danilchanka O, Pavlenok M, Niederweis M: Role of porins for uptake of antibiotics by Mycobacterium smegmatis. Antimicrob Agents Chemother 2008, 52:3।27-3I34.

31. Hillmann D, Eschenbacher I, Thiel A, Niederweis M: Expression of the major porin gene mspA is regulated in Mycobacterium smegmatis. J Bacteriol 2007, I89:958-967.

32. Molle V, Saint N, Campagna S, Kremer L, Lea E, Draper P, Molle G: pH-dependent pore-forming activity of OmpATb from Mycobacterium tuberculosis and characterization of the channel by peptidic dissection. Mol Microbiol 2006, 61:826-837.

33. Raynaud C, Papavinasasundaram KG, Speight RA, Springer B, Sander $\mathrm{P}$, Bottger EC, Colston MJ, Draper P: The functions of OmpATb, a pore-forming protein of Mycobacterium tuberculosis. Mol Microbiol 2002, 46:191-201.

34. Brosch R, Pym AS, Gordon SV, Cole ST: The evolution of mycobacterial pathogenicity: clues from comparative genomics. Trends Microbiol 200I, 9:452-458

35. Sambrook J, Russell DW: Molecular Cloning - A Laboratory Manual. Third edition. New York, U.S.: Cold Spring Harbor Laboratory Press; 2001 .

36. Lewin A, Freytag B, Meister B, Sharbati-Tehrani S, Schafer H, Appel B: Use of a quantitative TaqMan-PCR for the fast quantification of mycobacteria in broth culture, eukaryotic cell culture and tissue. J Vet Med B Infect Dis Vet Public Health 2003, 50:505-509.

37. Hanahan D: Studies on transformation of Escherichia coli with plasmids. I Mol Biol 1983, 166(4):557-580.

38. Kimura M: A simple method for estimating evolutionary rates of base substitutions through comparative studies of nucleotide sequences. J Mol Evol 1980, 16:1 II-120.

39. Strauch E, Voigt I, Broll H, Appel B: Use of a plasmid of a yersinia enterocolitica biogroup IA strain for the construction of cloning vectors. J Biotechnol 2000, 79:63-72.

40. Stover CK, de la Cruz VF, Fuerst TR, Burlein JE, Benson LA, Bennett LT, Bansal GP, Young JF, Lee MH, Hatfull GF: New use of BCG for recombinant vaccines. Nature 199I, 35 I:456-460.

41. Bashyam MD, Tyagi A: An efficient and high-yielding method for isolation of RNA from mycobacteria. Biotechniques 1994, I 7:834-836.

42. Sander P, Meier A, Bottger EC: rpsL+: a dominant selectable marker for gene replacement in mycobacteria. Mol Microbiol 1995, 16:991-1000.

43. Wiles S, Ferguson K, Stefanidou M, Young DB, Robertson BD: Alternative Luciferase for Monitoring Bacterial Cells under Adverse Conditions. Appl Environ Microbiol 2005, 71:3427-3432.

Publish with Bio Med Central and every scientist can read your work free of charge

"BioMed Central will be the most significant development for disseminating the results of biomedical research in our lifetime. "

Sir Paul Nurse, Cancer Research UK

Your research papers will be:

- available free of charge to the entire biomedical community

- peer reviewed and published immediately upon acceptance

- cited in PubMed and archived on PubMed Central

- yours - you keep the copyright 